\title{
Wissenschaft(sgeschichte) im Kinderkrimi
}

\author{
Beate Ceranski
}

Bellinda, 2006. Der gestohlene Geigenkasten. Ein Ratekrimi um Albert Einstein. Bindlach: Loewe Verlag, geb. 128 S., 7,90 €, ISBN-13: 978-3-7855-5758-7.

Bellinda, 2008. Bellinda, 2008. Verrat unterm Sternenhimmel. Ein Ratekrimi um Galileo Galilei. Bindlach: Loewe Verlag, geb. 128 S., 7,90 €, ISBN-13: 978-3-7855-4561-4.

Bellinda, 2009. Alarm im Laboratorium. Ein Ratekrimi um Marie Curie. Bindlach: Loewe Verlag, geb. 128 S., 7, $90 €$, ISBN-13: 978-3-7855-6377-9.

Neubauer, Annette, 2006. Ein Fall für den Meisterschüler. Ein Ratekrimi um Leonardo da Vinci. Bindlach: Loewe Verlag, geb. 128 S., 7,90 €, ISBN-13: 978-3-7855-5882-9.

Neubauer, Annette, 2007. Anschlag auf die Buchwerkstatt. Ein Ratekrimi um Johannes Gutenberg. Bindlach: Loewe Verlag, geb. 128 S., 7,90 €, ISBN-13: 978-3-78554562-1.

Neubauer, Annette, 2009. Im Bann der Alchemie. Ein Ratekrimi um Isaac Newton. Bindlach: Loewe Verlag, geb. 128 S., 7,90 €, ISBN-13: 978-3-7855-6609-1.

Rothballer, Michael, 2007. Das Geheimnis der Dracheninsel. Ein Ratekrimi um Charles Darwin. Bindlach: Loewe Verlag, geb. 128 S., 7,90 €, ISBN-13: 978-3-7855-5923-9. 
Rübenstrunk, Gerd, 2008. Explosion in der Motorenhalle. Ein Ratekrimi um Rudolf Diesel. Bindlach: Loewe Verlag, geb. 128 S., 7,90 €, ISBN-13: 978-3-7855-5926-0.

Die hier zu besprechenden Ratekrimis für Kinder dürften dem ersten Eindruck nach für die Leserinnen und Leser von NTM weniger als eigene Dienstlektüre denn als Geschenktipp von Interesse sein. Wo Papiere von Albert Einstein verschwinden, Rudolf Diesels Motoren wiederholten Sabotageakten ausgesetzt sind oder auf Charles Darwins „Beagle“ zwielichtige Gestalten an Bord sind, da handelt es sich nicht um einen Aufsatz für eine Fachzeitschrift oder eine Forschungsmonographie. $\mathrm{Zu}$ lesen sind vielmehr fiktive Abenteuer historischer Akteurinnen und Akteure, die gleichwohl den Anspruch erheben, ihre junge Leserschaft an die jeweiligen historischen Themen heranzuführen.

Der Loewe-Verlag hat seine ersten wissenschaftshistorischen Ratekrimis im Juni 2006 auf den Markt gebracht. Die damit eröffnete Reihe „Tatort Forschung" folgte der einige Jahre älteren $\mathrm{zu}$ allgemeinhistorischen Themen, die unter dem Titel „Tatort Geschichte" sehr gut am Markt angekommen war. Bei ihr hatte sich die Themenauswahl ganz pragmatisch am gymnasialen Lehrplan Geschichte orientiert, der für die anvisierte Altersgruppe in den Klassenstufen 5 und 6 Antike und Mittelalter vorsieht. Für die neue Reihe, die bei den Kindern Interesse für die Naturwissenschaften wecken soll, gab es eine solcherart curricular vorgegebene Leitlinie nicht. Anders als die meist themenzentrierten allgemeinhistorischen Ratekrimis sind sie ausschließlich an Personen ausgerichtet. Den Einstieg bildeten ein Band um Albert Einstein und eine Geschichte um Leonardo da Vinci. Bis heute sind insgesamt neun Titel erschienen. Die Reihe wird weiter fortgesetzt, jedoch inzwischen im halben Tempo mit zwei Bänden pro Jahr; der jüngste beschäftigt sich unter dem Titel „Im Bann der Alchemie“ mit Isaac Newton. Inzwischen ist in zwei Fällen auch eine Taschenbuchausgabe für fünf Euro erschienen, die nicht zuletzt die Auswahl der Geschichten als Klassenlektüre ermöglichen soll.

Inhaltlich handelt es sich um eine typische Buchreihe für Kinder in dem Sinn, dass die Bände einem einheitlichen Strickmuster folgen. Der jeweiligen wissenschaftshistorischen Hauptfigur widerfahren mysteriöse Dinge, beispielsweise der Diebstahl eines wichtigen Dokuments, die von Kindern aus ihrem Umfeld aufgeklärt werden. Die Kinderfiguren sind oft fiktiv, aber es gibt auch Fälle, in denen Sohn oder Tochter der Hauptperson als Detektivfigur fungieren (z. B. Curie und Diesel). Die Kinder treten 
stets als gemischtes Doppel oder Dreiergruppe auf, so dass mit diesem Kunstgriff Mädchen und Jungen gleichermaßen angesprochen werden. Die Leserinnen und Leser sind nun angehalten, gemeinsam mit den kindlichen Detektivfiguren dem drohenden oder begangenen Unheil auf die Schliche zu kommen und den Bösewicht zu entlarven. Die entscheidenden Hinweise erhalten sie stets in einer Illustration, wobei jede Rateaufgabe eine andere Schwierigkeit enthält: das Entziffern einer verschlüsselten Botschaft, Entdecken eines verräterischen Sachdetails, das Verfolgen einer Route auf einem Stadtplan und anderes mehr. Die Illustrationen spielen demnach für den Plot eine zentrale Rolle und prägen auch sonst das Gesicht der Bücher. Dementsprechend schnell sind von hungrigen Lesemäulern die 128 Seiten pro Band verknuspert, zumal davon nur gut hundert auf die eigentliche, durchweg flott und gut geschriebene Krimigeschichte entfallen. Ergänzt werden sie durch weiterführende Informationsmaterialien wie Zeittafeln, Glossare für fremd(sprachig)e und Fachwörter sowie Kurzbiographien beziehungsweise kurze Darstellungen der Forschungen der betreffenden Wissenschaftlerfigur.

Aus wissenschaftshistorischer Perspektive sind die Bände durchweg gelungen, und man kann davon ausgehen, dass sich die Autorinnen und Autoren - die oft auch noch in anderen Themenbereichen für den Verlag geschrieben haben - anhand einschlägiger Literatur eingearbeitet haben. Die Geschichten sind zeitlich auf dem Höhepunkt der jeweiligen Schaffensphasen angesiedelt und kreisen, dem kriminalistischen Genre nach, oft um Anfeindungen, denen die Forschenden ausgesetzt waren. Nur im Darwin-Band, während der Forschungsreise spielend, gibt es keinen Konflikt des Wissenschaftlers mit seiner Umgebung. Die dem wissenschaftshistorischen Handlungsstrang fernliegende Schatzsuche auf einer der Galapagos-Inseln hält von allen hier vorgestellten Büchern die größte Distanz zur wissenschaftlichen beziehungsweise wissenschaftshistorischen Thematik; die Figur Darwins wäre gegen jeden anderen Forschungsreisenden austauschbar. Im Einstein-Band, der 1920 in Berlin spielt, blitzen hingegen die antirelativistischen Umtriebe als Tatmotiv auf, bei Marie Curie wird der Widerstand gegen ihre Aufnahme in die Académie des Sciences ausgemalt, bei Diesel sind es die Patentstreitigkeiten und bei Galilei natürlich der Konflikt mit kirchlichen Kreisen seiner Zeit. Dass es in all diesen Biographien Konfliktstoff genug gab, ist für Leserinnen und Leser der NTM keine Überraschung, aber die Reihe bietet tatsächlich einen ganz reizvollen Anlass, über die Typen und Muster von Konflikten nachzudenken, 
die Wissenschaftler erlebten. Aus wissenschaftshistorischer Sicht ist dabei bemerkenswert, dass gerade der zeitlich sehr klug angelegte Curie-Band, der die Forscherin im Kontext ihrer Akademiekandidatur zeigt, ein Thema stark macht, das von der jüngeren Wissenschaftsgeschichtsschreibung inzwischen deutlich relativiert wurde, nämlich den edelmütigen Verzicht der Curies auf die Patentierung ihrer Forschungsergebnisse. Offensichtlich strahlt hier das von Tochter Eve gezeichnete Lebensbild der Wissenschaftlerin immer noch so stark aus, dass die - oft nur fremdsprachig oder in der Fachliteratur zugänglichen - Relativierungen dieses Bildes in der neueren Forschung darüber einfach nicht wahrgenommen werden.

Die ergänzenden Materialien bieten für Kinder verständliche, aber dennoch zutreffende Erklärungen an, wobei man freilich an ihre Tiefe nicht allzu hohe Ansprüche stellen sollte. Unangenehm fällt hier das Glossar des Einstein-Buches auf, in dem es bei der Erklärung der berühmten Einstein'schen Energie-Masse-Äquivalenz zu der physikalisch, gelinde gesagt, außerordentlich unglücklichen Formulierung kommt: „Masse kann in eine enorme Energie umgewandelt werden, wenn sie mit dem Quadrat der Lichtgeschwindigkeit multipliziert wird." (Bellinda 2006: 118) Hier gab es offensichtlich kein naturwissenschaftlich kompetentes Lektorat mehr.

Neben den Informationen über Leben und Forschungen der jeweiligen Wissenschaftlerinnen und Wissenschaftler werden auch viele kulturgeschichtliche Details behandelt, wobei die Balance nicht immer ganz glücklich ist. Der Galilei-Band etwa ist voll gespickt mit italienischen Wendungen und Begriffen, die zwar alle im Glossar erläutert werden, aber die Konzentration beim Lesen doch mitunter $\mathrm{zu}$ Lasten der inhaltlichen Hauptlinien $\mathrm{zu}$ sehr in Anspruch nehmen. Andererseits aber liegt in den kulturgeschichtlichen Aufbereitungen durchaus auch eine Stärke; der Band um Einstein etwa bietet Einblicke in Haushaltsstruktur und Ernährungsgewohnheiten des jüdischen Großbürgertums in Berlin um 1920.

Als Fazit ist demnach der äußerst erfreuliche Befund festzuhalten, dass mit „Tatort Forschung“ eine Buchreihe auf dem Markt ist, die Kindern im Übergangsalter zur Sekundarschule einen für sie attraktiven und meistens sachlich kompetent aufbereiteten Zugang zu historischen Entwicklungen aus Naturwissenschaften und Technik bietet. Aus eigener Elternerfahrung ist auch zu berichten, dass ein solcher Ratekrimi tatsächlich den Einstieg in eine vertiefte Beschäftigung mit einem wissenschaftshistorischen Thema im schulischen Kontext motivieren kann. 
Einmal mehr ist damit zu konstatieren, was auch in anderen Kontexten bereits formuliert wurde: Trotz der hochschulpolitisch oft ungünstigen Entwicklungen, die die Wissenschaftsgeschichte in den letzten Jahren viele Professuren gekostet hat, hat das Fach in der öffentlichen Wahrnehmung ganz klar Karriere gemacht. Damit wäre es aber, so meine ich, an der Zeit, auch aus unserer Sicht diese Entwicklung wahrzunehmen, zu reflektieren und auf mögliche Konsequenzen für uns zu befragen. In der Geschichtswissenschaft ist die Reflexion der historischen Popularisierung inzwischen als Thema breit angekommen und reicht in ihren Analysen, bezeichnenderweise, inzwischen bis in das Genre des Krimis hinein. Für die Wissenschaftsgeschichte hingegen ist mir, bis auf vereinzelte Ausnahmen etwa in einer wissenschaftshistorischen Reflexion in einem Sammelband zu Michael Frayns Kopenhagen, gerade in der deutschsprachigen Landschaft kaum eine Auseinandersetzung mit der Popularisierung wissenschaftshistorischer Themen, sei es in der ,trivialen' oder ,klassischen' Literatur, bekannt. Schließlich bleibt festzuhalten, dass gerade in der populären Rezeption kein Weg an einer intensiven Personalisierung der Wissenschaftsgeschichte vorbeizuführen scheint. Für unser Fach, das schon seit vielen Jahren keine, Geschichten großer Männer' mehr schreiben will, bleibt dies eine besondere Herausforderung. Sollten wir uns ihr nicht stellen - und dies nicht nur analytisch, sondern auch durch eigene Darstellungen? Damit es auch weiterhin attraktive und angemessene wissenschaftshistorische Bücher für Kinder und ein großes Publikum gibt!

Beate Ceranski

Geschichte der Naturwissenschaften und Technik

Universität Stuttgart

Keplerstr. 17

D-70174 Stuttgart, Deutschland

E-Mail: Beate.Ceranski@po.hi.uni-stuttgart.de 